\title{
Inlet Channel Effects on Archimedes Screw Generators
}

\author{
Adil Khan, Scott Simmons, Murray Lyons, William Lubitz ${ }^{1}$ \\ School of Engineering \\ University of Guelph \\ Guelph, Ontario, Canada \\ ${ }^{1}$ wlubitz@uoguelph.ca
}

\begin{abstract}
The Archimedes screw is one of the oldest machines in the world, and has been used for various applications including hydroelectric power generation. This project investigated the effect of inlet conditions on a laboratory-scale Archimedes screw as a generator. The performance of the Archimedes screw was measured with both a narrow inlet channel and a wider inlet basin. The required inlet water depth was experimentally determined for both the channel and the basin, for a range of operating flow rates and screw rotation speeds. The tests with and without the channel were conducted at 9 different speeds and 9 different flows. The data was analyzed and plotted for efficiency, head, inlet depth, nondimensional inlet depth with the respective flow and speed associated to each data point. The experimental data was used to develop an inlet depth model. The data was analyzed and plotted for efficiency, head, inlet depth, non-dimensional inlet depth with the respective flow and speed associated to each data point. It was found that there was a notable efficiency difference between the basin and channel inlets only at low volume flow rates and high rotation speeds.
\end{abstract}

Keywords-Archimedes screw; inlet channel; efficiency; head

\section{INTRODUCTION}

The Archimedes screw is an ancient machine that has been used continually for millennia. Initially the Archimedes screw was used for drainage and irrigation purposes [1]. Applications of the Archimedes screw today include, but are not limited to, wastewater treatment plant pumps, low-lying land pumping stations, irrigation systems, fish conveyors, and generators [2].

An Archimedes screw consists of a set of helical planes, or flights, which are connected to a cylindrical central shaft, and supported on bearings that allow it turn within a close-fitting cylindrical trough or enclosure [3]. The design of the Archimedes screw is such that when the screw is tilted, the blades of the screw trap water discrete volumes of water called buckets [1]. The rotation of the screw allows the water to travel within the blades. The Archimedes screw can be used either as a pump or a generator. When water is moving from the bottom to the top, work is done turning the screw and it is being used as a pump. When water is moving from the top to the bottom, work is done by the rotating Archimedes screw and it can be used to turn a generator to produce electricity.
In the 1930's there were over 300 Archimedes screws in the Netherlands being used as drainage pumps in low-lying regions [4]. Use of the Archimedes screw for power generation began with experimental tests conducted at the University of Prague between 1993 and 1995 by Brada [2]. These experiments demonstrated that an Archimedes screw used for power generation could have more than $80 \%$ efficient converting hydraulic energy into mechanical energy [2]. It has been shown and proven that better conversion efficiencies can be attained by using larger screws as per Hellmann and Kleemann [2].

Currently over 400 Archimedes screw generators (ASGs) have been installed in Europe, with the first being installed only in 1993 [5]. Archimedes screw generators remain rare outside Europe. The first connection of an Archimedes screw generator to a power grid in North America was in 2013 by Greenbug Energy near Waterford, Ontario, Canada [5]. Unlike most hydro turbines, fish can pass through an operating Archimedes screw unharmed [5]. Additional advantages of Archimedes screws include straightforward design, good efficiency at non-optimum flows, ease of use, and low environmental impacts [6].

Water for an ASG is typically supplied by an inlet channel that conveys water from a reservoir or other source to the inlet of the screw. During design of an ASG installation, it is essential to ensure that the water depth that can be supplied at the inlet to the screw will be sufficient to provide the desired flow rate and amount of filling once the water enters the screw. If the inlet basin level is too low, for example, it will not be possible to fill the buckets in the operating screw.

The required inlet water level will be a function of both the screw rotation speed and the volume flow rate of water to be supplied. For example, if the screw is turning very quickly, there will be insufficient time to fill each bucket of the operating screw, and the screw will be unable to produce the desired power.

The goal of this project was to experimentally investigate the impact of volume flow rate and screw rotation speed on the inlet basin water depth. Two cases were tested to check the effect of inlet channel geometry on this relationship: one with a narrow inlet channel and the other with a much wider

A range of cross-sections can be used in an inlet channel. The channel used for this experiment had a rectangular cross-section, with a flat bottom and vertical, parallel side walls. 
The project involved the use of an inlet channel for the testing to develop a relationship of a non-dimensional model which can predict head, flow, and speed. The outlet channel losses were held constant for all tests during the experiment because the depth of the water at the screw outlet was always below the screw in the lower basin. The inlet channel losses depend upon the depth of water entering the screw and leaving the screw. It should be noted that the Borda-Carnot entrance loss technique can be used for calculating the inlet channel head loss and has been done by [2]. Kozyn found that the actual power loss at the entrance was small relative to other losses in an ASG.

This project involved the construction of a rectangular cross section channel which was connected to the Archimedes screw generator. This connecting channel was used to develop relationships with respect to the flow, head, torque, and speed of an Archimedes screw generator. The objective of the project was to determine the required water depth to achieve a specific volume flow rate through the Archimedes screw generator over a range of screw speeds within the operating range of the Archimedes screw generator.

\section{EXPERIMENTAL METHODOLOGY}

Experiments were conducted at the University of Guelph Archimedes screw testing facility with, and without the rectangular inlet channel installed within the upper supply basin to the Archimedes screw. Tests were conducted on a threebladed screw with a $31.6 \mathrm{~cm}$ outer diameter, $16.8 \mathrm{~cm}$ inner cylinder diameter, $31.8 \mathrm{~cm}$ pitch and $122 \mathrm{~cm}$ flighted length

The setup of the testing facility involved an upper basin that fed the Archimedes screw with water and that water would output to a lower basin (Fig. 1). The water from the lower basin was fed to the upper basin through a pipe. There was a pump which allowed for the water to flow through the pipe to get to the upper basin. Upon completion of the experiments with just the Archimedes screw, a rectangular cross-section inlet channel was designed with width of $31.8 \mathrm{~cm}$, length of $122 \mathrm{~cm}$ from upstream channel entrance to screw entrance. The sides of the channel entrance were designed with a radius of $2.5 \mathrm{~cm}$ to reduce channel entrance losses. The floor of the channel was aligned with the bottom-most edge of the screw inlet. This channel was machined by a local machining company in Guelph and installed in the Archimedes screw testing facility. The channel was installed and then tests were conducted at the 9 different flows and 9 different rotation speeds. The flows and speeds that were tested were the same with and without the channel in place.

The screw was operated at an inclination angle $\beta$ of $24.5^{\circ}$, at rotation speeds of RPM, 25 RPM, 30 RPM, 35 RPM, 40 RPM, 50 RPM, 60 RPM, 70 RPM, and 80 RPM. At each rotation speed, torque and inlet basin depth were recorded for flows of 6 $\mathrm{L} / \mathrm{s}$ to $14 \mathrm{~L} / \mathrm{s}$ in one L/s increments. These ranges of speed and flow were chosen to provide a range of results across the performance envelope of the screw.

The Archimedes screw was operated in generator mode and the instruments which were available in the University of Guelph Archimedes screw testing facility were used for the measurements of flow, head, torque, and speed $[3,8]$.

The shaft power generated by the screw $P$ was calculated from the angular rotation speed of the screw $\omega$ and torque $T$ :

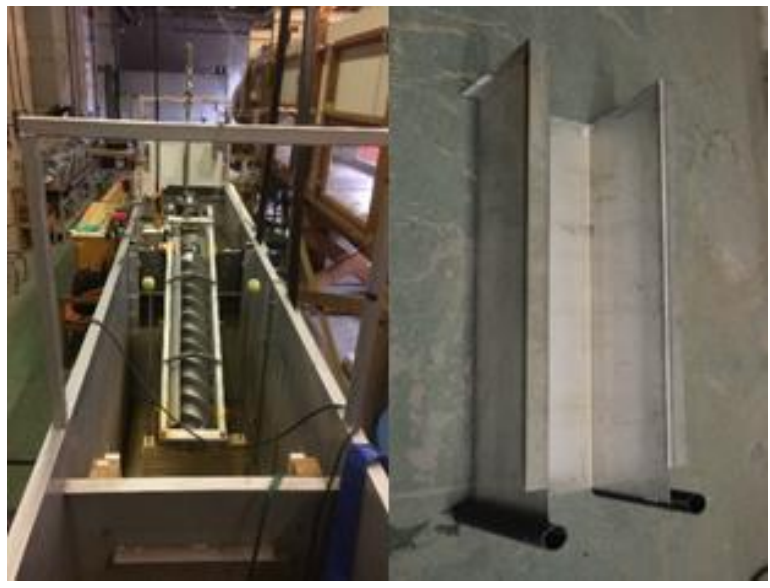

Figure 1. Archimedes screw (left) and inlet channel (right).

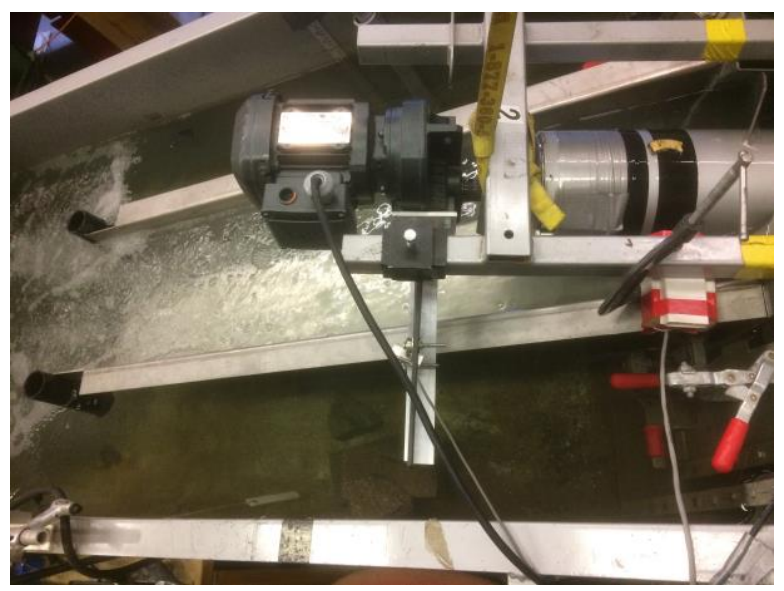

Figure 2. Upper basin with channel installed, with top of screw frame including gear motor and torque arm.

$$
P=\omega T
$$

The rotational speed of the screw was measured with a magnetic switch which was positioned just above the shaft at the inlet of the screw. A magnet secured on the screw shaft caused the magnetic switch to close once revolution [3]. The switch state was recorded by a National Instruments (NI) USB-6009 Data Acquisition (DAQ) unit connected to a laptop computer running NI LabVIEW. The magnetic switch data was recorded at a frequency of $1000 \mathrm{~Hz}$.

It should be noted that ASGs rotate relatively slowly. In practice, most full-scale ASGs used for power generator rotate at a speed close to

$$
\omega_{m}=\frac{5 \pi}{3 D_{o}^{2 / 3}}
$$

where $D_{0}$ is the screw outer diameter in meters and $\omega_{m}$ is rotation speed in radians per second.

Screw torque was measured by an Omegadyne LC703-25 load cell with one end fixed to an arm attached to a variablefrequency drive that regulated rotation speed, and the other end fixed to a stationary point on the support frame of the screw [8]. 
Efficiency vs Speed at $6 \mathrm{~L} / \mathrm{s}$

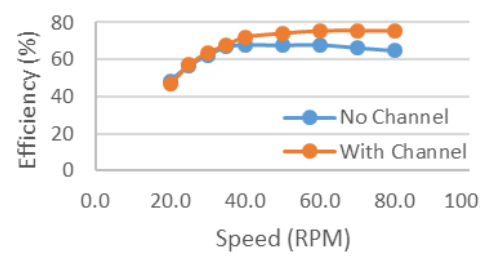

Efficiency vs Speed at $9 \mathrm{~L} / \mathrm{s}$

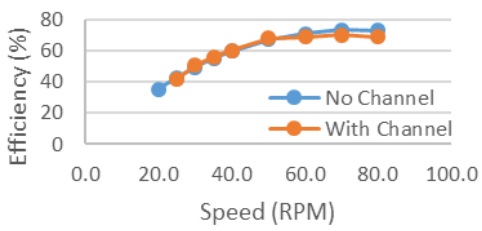

Efficiency vs Speed at $12 \mathrm{~L} / \mathrm{s}$

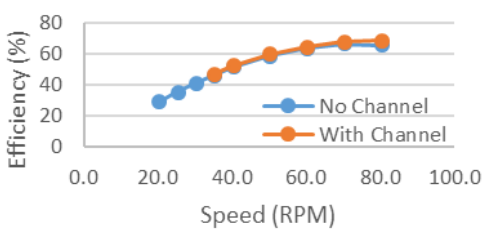

Efficiency vs Speed at $7 \mathrm{~L} / \mathrm{s}$

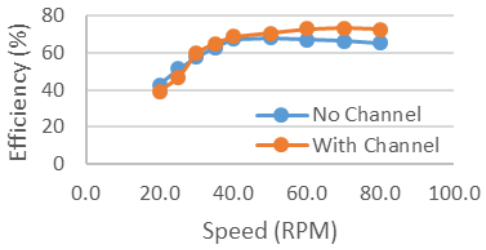

Efficiency vs Speed at $10 \mathrm{~L} / \mathrm{s}$

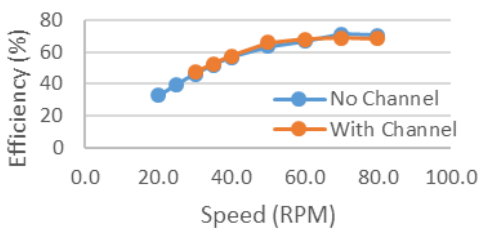

Efficiency vs Speed at $13 \mathrm{~L} / \mathrm{s}$

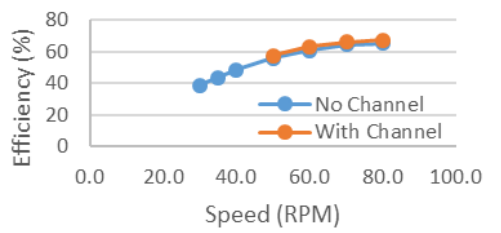

Efficiency vs Speed at $8 \mathrm{~L} / \mathrm{s}$

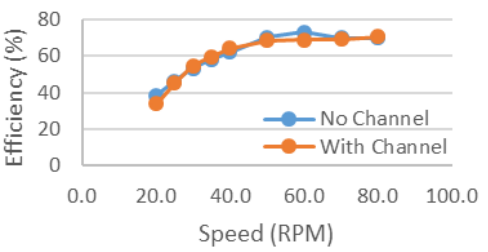

Efficiency vs Speed at $11 \mathrm{~L} / \mathrm{s}$

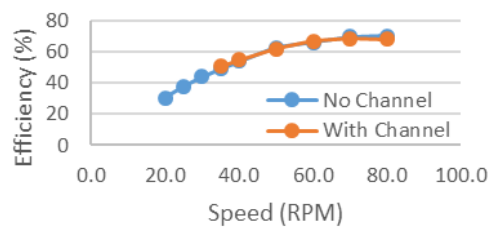

Efficiency vs Speed at $14 \mathrm{~L} / \mathrm{s}$

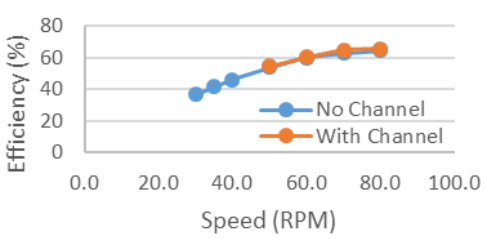

Figure 4. Efficiency vs Speed at Specified Flows

The load cell was installed so that the line of action through the cell was $26.5 \mathrm{~cm}$ from the screw axis of rotation.

The maximum power available to the screw is

$$
P_{\max }=\rho g h Q
$$

where $\rho$ is water density, $g$ is the gravitational constant $(9.81$ $\left.\mathrm{m} / \mathrm{s}^{2}\right), h$ is the height difference between the free surfaces in the upper and lower basin, and $Q$ is volume flow rate of water. The screw was operated with the receiving basin water level below the outlet of the screw to ensure consistence, so $h$ was taken as the vertical distance from the inlet basin free surface, to the lowermost point on the screw outlet. The heights of the screw as tested are shown in Fig. 3. The efficiency of the screw is then

$$
\eta=\frac{P}{P_{\max }}
$$

The water level in the inlet basin was measured using a Keller Valueline depth gauge and visually confirmed. Volume flow rate was measured with an Omega FTB740 flow meter

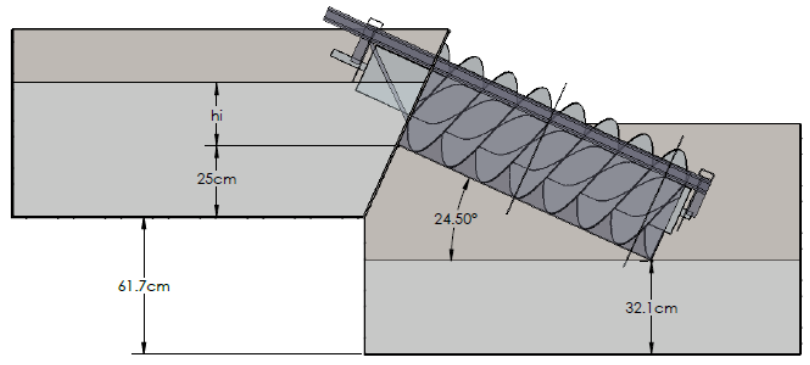

Figure 3. Heads and vertical distances as tested. installed in the return pipe that through which water is pumped from the lower basin back to the inlet basin. All flow through the system also passes through the screw, so this measurement also reflects the flow rate through the screw.

Non-dimensional inlet depth was calculated as

$$
\zeta=\frac{h_{i}}{D_{o} \cos \beta}
$$

where $h_{i}$ is vertical distance between the inlet basin free surface, and the lowest point of the screw inlet opening (Fig. 3).

\section{ERROR ANALYSIS}

An error analysis was performed in which observed sensor errors and uncertainties were propagated through the calculations to determine the uncertainty of calculated efficiency and power. Power generated by the screw is the product of torque and angular velocity. The magnetic switch observing rotation was recorded at a frequency of $1000 \mathrm{~Hz}$, and rotation rate was based on the precise time between the series of full rotations within the one minute sampling period. This allowed for the uncertainty in angular velocity to be negligible relative to the torque measurement [3]. The uncertainty for the torque measurements was previously found to be $0.22 \mathrm{Nm}$ [8]. The overall result was that under nominal conditions, the uncertainty of the calculated power was $\pm 1.0 \mathrm{~W}$ and efficiency uncertainty at nominal conditions is \pm 0.015 .

\section{RESUlTS AND DisSUCSION}

The effect of supplying water to the screw through a narrow inlet channel, rather than directly from a wide supply basin, was 
relatively minor. Figs. 4 and 5 show that across much of the tested range, the efficiency of the screw was effectively the same whether it was operating with a narrow inlet channel or the wider inlet basin. The effect of the inlet channel only becomes apparent at low volume flow rates and higher rotational speeds. Above 40 RPM the efficiency of the screw is higher with the channel then without the channel for $6 \mathrm{~L} / \mathrm{s}$. A similar trend occurred at $7 \mathrm{~L} / \mathrm{s}$, while at all higher flow rates there were no significant differences in efficiency given the uncertainty in the calculated efficiency.

Interestingly, in low flow, high rotation conditions, the efficiency of the screw was higher with the narrow channel in place than it was without the channel. This was not expected: it was anticipated that the presence of the channel would result in decreased overall efficiency. The overall head across the screw is measured in the upstream area of the basin, and so any channel losses with the inlet channel would be expected to produce additional head loss, reducing system efficiency relative to the case of operating with the screw being supplied directly from the wide inlet basin.

It was determined that the efficiency difference was due to entrance effects when water enters the screw. Figs. 6 to 9 show the water surface at the entrance to the operating Archimedes screw. With the channel in place there is a relatively linear surface, and flow is relatively uniform into the screw. Without the channel, water enters the screw from the wide basin from both the front and sides of the inlet. Fig. 10 shows a notably variation in water level at the entrance, due to the flow from the basin sides turning around the sharp edged inlet and into the screw entrance. This was seen for both the $6 \mathrm{~L} / \mathrm{s}$ and $7 \mathrm{~L} / \mathrm{s}$ flows. This additional entrance loss without the channel in place results in an increased head loss. At higher flow rates, there was little apparent difference in the water surface at the entrance with and without the channel in place.

Note that the data point with $8 \mathrm{~L} / \mathrm{s}$ and 60 RPM may be subject to experimental error. It is believed that some air was drawn by the pump into the return pipe while this data point was taken, causing the flow meter to erroneously measure a slightly lower-than-actual flow.

Considering inlet depth generally (with or without the narrow inlet channel present), it was found that higher inlet basin depth was required in order to increase flow rates or decrease rotation speeds. Fig. 5 graphically shows this relationship.

Simple models of inlet depth were developed by first noting that screw rotation speed and volume flow rate would be the dominant dependent variables:

$$
\begin{aligned}
h_{i} & =f(\omega, Q) \\
\zeta & =f\left(\frac{\omega}{\omega_{m}}, \frac{Q}{Q_{m}}\right)
\end{aligned}
$$

where

$$
Q_{m}=\left[\frac{\pi}{4}\left(D_{o}{ }^{2}-D_{i}^{2}\right)\right]\left[\frac{S \omega}{2 \pi}\right]
$$

is a theoretical rate at which material would pass through the full cylindrical volume of the screw (excluding the inner cylinder) due to screw rotation only.
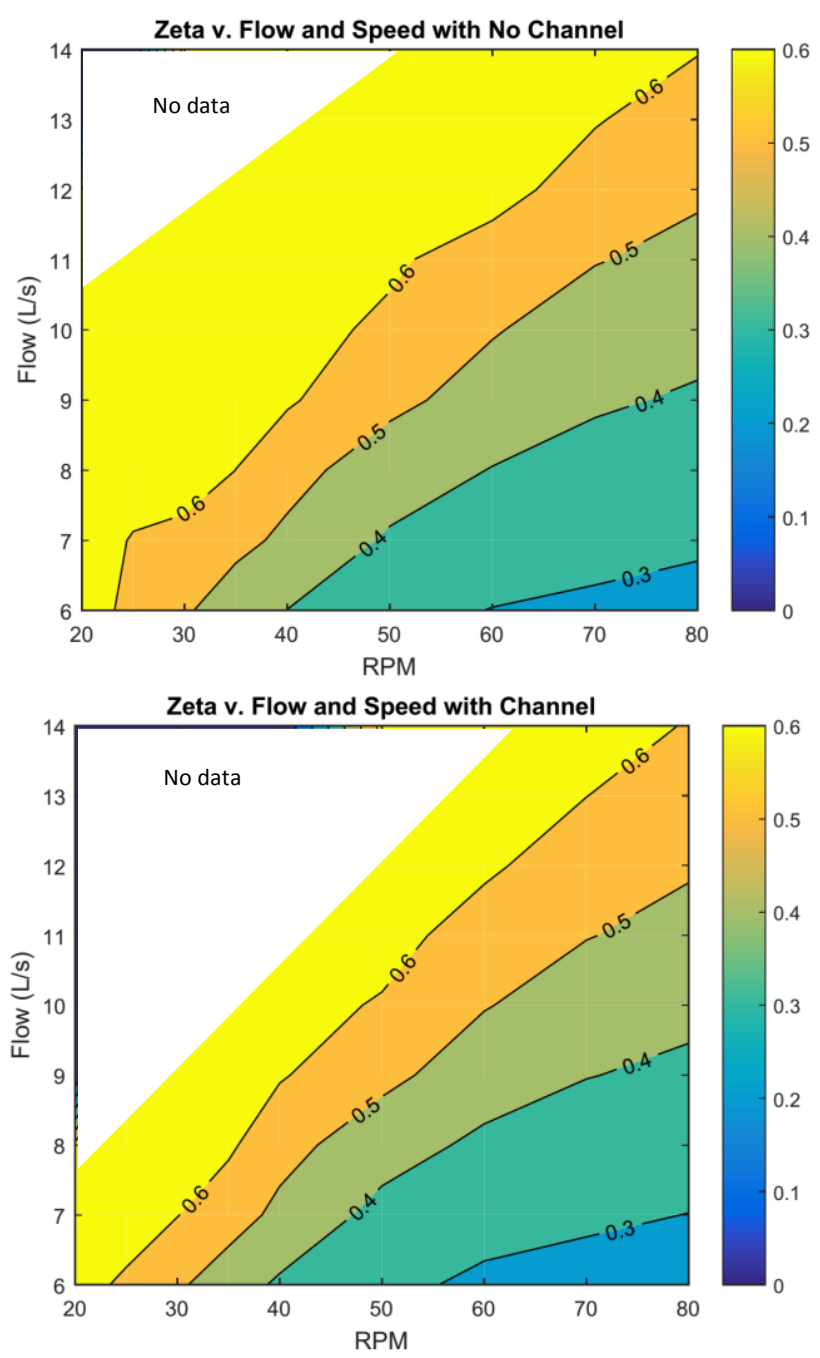

Figure 5. Contours of non-dimensional inlet depth vs. flow and rotation speed without channel installed (top) and with channel in place (bottom)

Simple predictive models were then developed by fitting the measured data (e.g. Fig. 4) to Eqns. 6 and 7. The resulting model for the inlet depth in dimensional form is

$$
h_{i}=\frac{Q}{\left[15.51 \mathrm{~L}^{1} \mathrm{~m}^{-1} \mathrm{rad}^{-1}\right] \times \omega}
$$

This equation gives a fairly accurate representation of the inlet depth with a mean absolute error of 3.3\%. Similarly, a nondimensionalized relationship was found as

$$
\zeta=\frac{\omega Q}{6.84\left(\omega_{m} Q_{m}\right)}
$$

The equation developed for the non-dimensional inlet depth with respect to the flow and speed was found by trial and error. This equation gives a representation of the non-dimensional inlet depth with a mean absolute error of $9.4 \%$.

It should be noted that scaling between lab ASGs and full scale ASGs has been identified as an issue in need of further study [3]. While Eqn. 10 is cast non-dimensionally, it should be noted that entrance Reynolds number and Froude number will 


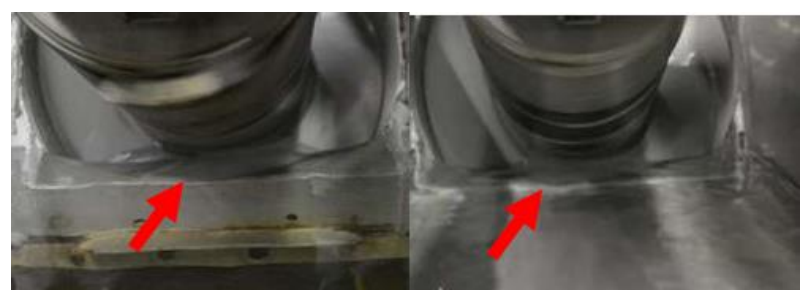

Figure 6. Flow of $6 \mathrm{~L} / \mathrm{s}$ with no channel (left) and channel (right) at 80 RPM at the inlet of the screw

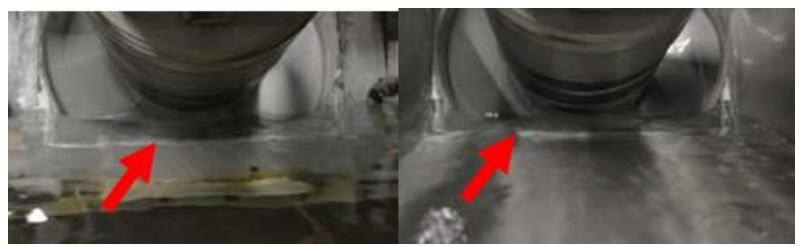

Figure 7. Flow of $7 \mathrm{~L} / \mathrm{s}$ with no channel (left) and channel (right) at 80 RPM at the inlet of the screw

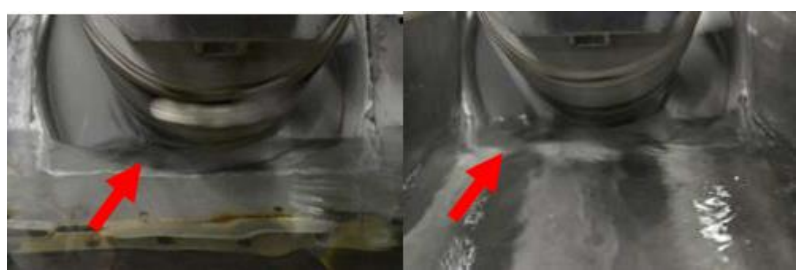

Figure 8. Flow of $8 \mathrm{~L} / \mathrm{s}$ with no channel (left) and channel (right) at 80 RPM at the inlet of the screw

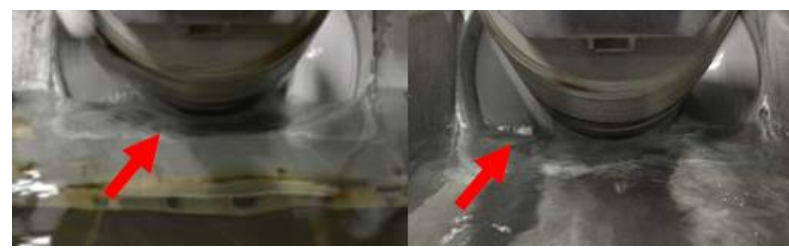

Figure 9. Flow of $9 \mathrm{~L} / \mathrm{s}$ with no channel (left) and channel (right) at 80 RPM at the inlet of the screw

change with ASG scale, and effects such as the criticality of the entrance flow are not included in this simple model. Therefore, Eqn. 10 should be used with caution in differing situations.

\section{CONCLUSION}

The Archimedes screw is a practical device that has been used for many years with various applications. There are still many things to learn about the Archimedes screw. This project investigated the inlet water depth to required achieve a specific volume flow rate through the Archimedes screw generator over a range of screw speeds. Tests were conducted with both a narrow inlet channel and wider inlet basin at the entrance to the screw. The measured efficiency was higher with the channel than without the channel for the $6 \mathrm{~L} / \mathrm{s}$ and $7 \mathrm{~L} / \mathrm{s}$ cases above 40 RPM. Entrance effects at the screw were identified as a possible cause of the reduced efficiency without channel present. There can be other reasons which would require further research into this topic. Relationships were developed for both the dimensional and non-dimensional inlet depth required to provide a specified volume flow rate across a range of rotational speeds. Additional testing of these relationships with data from other Archimedes screws is needed to verify prediction accuracy and determine if the relationships are broadly applicable to all Archimedes screws.

\section{ACKNOWLEDGMENTS}

The work documented in this study was completed as part of a much larger project financially supported by Greenbug Energy Inc. and the Natural Sciences and Engineering Research Council (NSERC) of Canada through the Collaborative Research and Development (CRD) program.

\section{REFERENCES}

[1] C. Rorres, "The turn of the screw: Optimal design of an Archimedes screw". Journal of Hydraulic Engineering, 126(1), pp 72-80, 2000

[2] D. M. Nuernbergk and C. Rorres, "Analytical Model for Water Inflow of an Archimedes Screw Used in Hydropower Generation", Journal of Hydraulic Engineering, 139(2), pp 213-220, 2012

[3] A. Kozyn and W. D. Lubitz, "A power loss model for Archimedes screw generators", Renewable Energy, doi:10.1016/j.renene.2017.02.062

[4] G. Nagel, "Archimedean Screw Pump Handbook", Schwäbisch Gmünd: RITZ Pumpenfabrik OHG, pp 10-11, 1968

[5] W. D. Lubitz, M. Lyons, and S. Simmons, "Performance model of archimedes screw hydro turbines with variable fill level", Journal of Hydraulic Engineering, 140(10), 4014050. doi:10.1061/(ASCE)HY.19437900.0000922

[6] Greenbug Energy Inc, "What are the benefits of using Archimedes Screws over other technologies? - GreenBug Energy - micro hydro", 2017, GreenBug Energy - micro hydro. Retrieved 21 May 2017, from http://greenbugenergy.com/sp_faq/what-are-the-benefits-ofusingarchimedes-screws-over-other-technologies

[7] D. A. Chin, "Water-resources engineering" (Third ed.), Pearson Education, pp. 140-144, 2013

[8] K. Songin, "Experimental Analysis of Archimedes Screw Turbines", Masters Thesis, University of Guelph. August 2017. https://atrium.lib.uoguelph.ca/xmlui/bitstream/handle/10214/11481/Song in_Kathleen_201708_MASc.pdf?sequence=1 